\title{
THE UNDERLYING UNITY BETWEEN MAGNETOSTATICS, FLUID FLOW AND DEFORMATION OF SOLIDS
}

\author{
M.J. MarcinkowsKi \\ Department of Mechanical Engineering, University of Maryland \\ College Park, Maryland 20742, USA \\ (Received June 27, 1991)
}

The study of deformation in solids, fluid flow and magnetostatics are all shown to possess a common genesis. In particular, this seemingly disparate group of disciplines can be reduced to a study of line defects. For solids, these defects are termed dislocations, whereas in fluid flow and magnetostatics they take the form of fluid and magnetic vortices, respectively. The mathematical formulations of all three are basically identical. For example, the Burgers vector, dislocation density, stress, and elastic constants associated with solids are replaced by circulation, vorticity, density and momentum, respectively in fluids, which in turn are replaced by current, current density, permeability and induction, respectively in magnetostatics. It is then possible to use various surface distributions of such defects to represent any type of stress, velocity or magnetic field. In this way, an externally stressed solid is shown to be equivalent to flow through a pipe, which in turn is analogous to a solenoid. Still further, a stressed hole, flow about a solid body and the Meissner effect are all demonstrated to be mathematically equivalent. This equivalence is underscored in a more basic manner by expressing the elastic distortion, fluid velocity and magnetic field in terms of similar gauge transformations.

PACS numbers: $61.70 . \mathrm{Ga}, 03.40 . \mathrm{Gc}, 41.10 . \mathrm{Dq}$

\section{Introduction}

The analogies that exist between various physical phenomena have often allowed a deeper understanding of each to be obtained as would have occurred by their study in isolation. For example, Burgers' [1] pioneering formulation of dislocation theory was aided by its strong mathematical connections with fluid flow about vortices. In a similar manner, Kröner's [2] elegant extension of Burgers' work drew heavily on the close relationship between elastostatics and magnetostatics. A review of this latter approach has been given by de Wit [3]. 
It has also been demonstrated that any state of elastic deformation can be represented in terms of some suitable distribution of surface dislocations $[4,5]$. This concept has been recently extended to show that any state of fluid motion can be described by means of unique distributions of surface vortices [6]. The logical application of these ideas to magnetostatics leads to the conclusion that any type of magnetic field can be described in terms of various unique distributions of magnetic vortices. We are thus led to the remarkable conclusion that the mechanical, fluid and electromagnetic behavior of matter can be reduced to the study of various types of line defects, either dislocations or vortices. The purpose of the present study is to develop these analogies in greater detail and to show how they can be used to provide a deeper mathematical and physical insight into the behavior of matter than has hitherto been available. In order to assist in a clearer understanding of the physical concepts involved, the present treatment will be limited to the linearized theory.

\section{The nature of line defects}

Whereas the elastic distortion $\beta_{i j}$ can be written in terms of the gradient of the displacement $u_{i}$, fluid velocity $v_{i}$ and magnetic intensity $H_{i}$ may be expressed in terms of the velocity and magnetic potentials $\phi_{\mathrm{V}}$ and $\phi_{\mathrm{M}}$, respectively. In particular

$$
\begin{aligned}
& \beta_{i j}=\partial_{i} u_{j}, \\
& v_{i}=\partial_{i} \phi_{\mathrm{V}}, \\
& H_{i}=\partial_{i} \phi_{\mathrm{M}} .
\end{aligned}
$$

In coordinate-free notation these become

$$
\begin{aligned}
& \boldsymbol{\beta}=\nabla \boldsymbol{u}, \\
& \boldsymbol{v}=\nabla \phi_{\mathrm{V}}, \\
& \boldsymbol{H}=\nabla \phi_{\mathrm{M}} .
\end{aligned}
$$

The correspondence between the various quantities can be readily seen by reference to Table I.

Quantities such as stress $\sigma_{i j}$, momentum $P_{i}$, and induction $B_{i}$, can now be defined in terms of $\beta_{i j}, v_{i}$ and $H_{i}$, respectively by means of the following constitutive relations:

$$
\begin{aligned}
& \sigma_{i j}=c_{i j k \ell} \beta_{k \ell}, \\
& P_{i}=\rho v_{i}, \\
& B_{i}=\mu H_{i},
\end{aligned}
$$

where $c_{i j k \ell}, \rho$ and $\mu$ are the elastic constants, density and permeability, respectively. Suppose now that the divergences of the above quantities are set equal to zero, leading to

$$
\begin{aligned}
& \partial_{j} \sigma_{i j}=0, \\
& \partial_{i} P_{i}=0, \\
& \partial_{i} B_{i}=0 .
\end{aligned}
$$


TABLE I

Correspondence between solid mechanics, fluid mechanics and magnetostatics.

\begin{tabular}{c|c|c}
\hline \hline Solids & Fluids & Magnetostatics \\
\hline Elastic displacement, $u_{i}$ & Velocity potential, $\phi_{\mathrm{V}}$ & Magnetic potential, $\phi_{\mathrm{M}}$ \\
Elastic distortion, $\beta_{i j}$ & Velocity, $v_{i}$ & Magnetic intensity, $H_{i}$ \\
Dislocations & Fluid vortex & Magnetic vortex \\
Burgers vector, $b_{i}$ & Circulation, $\Gamma$ & Current, $I$ \\
Dislocation density, $\alpha_{i j}$ & Vorticity, $w_{i}$ & Current density, $J_{i}$ \\
Elastic constant, $c_{i j k \ell}$ & Density, $\rho$ & Permeability, $\mu$ \\
Stress, $\sigma_{i j}$ & Momentum, $P_{i}$ & Magnetic induction, $B_{i}$ \\
Stress function, $A_{i j}$ & Vector potential, $A_{\mathrm{V} i}$ & Vector potential, $A_{\mathrm{Mi}}$ \\
Stress flux, $\Phi_{i}$ & Momentum flux, $\Phi_{\mathrm{V}}$ & Magnetic flux, $\Phi_{\mathrm{M}}$ \\
Self energy, $E_{\mathrm{DS}}$ & Kinetic energy, $E_{\mathrm{VS}}$ & Magnetic energy, $E_{\mathrm{MI}}$ \\
Interaction energy, $E_{\mathrm{DI}}$ & Interaction energy, $E_{\mathrm{VI}}$ & Interaction energy, $E_{\mathrm{MS}}$ \\
Peach-Koehler force, $F_{\mathrm{D}}$ & Magnus effect, $F_{\mathrm{V}}$ & Ampère's law, $F_{\mathrm{M}}$ \\
Externally stressed body & Pipe flow & Solenoid \\
Stressed hole & Flow around body & Meissner effect \\
Crack & Velocity concentration & Magnetic concentration \\
Elastic deformation & Irrotational flow & Irrotational field \\
Plastic deformation & Rotational flow & Rotational field
\end{tabular}

Equations (2.10), (2.11) and (2.12) insure that no sources or sinks of distortion, fluid flow or magnetism, respectively, exist. Alternatively, (2.10) is the condition of equilibrium, whereas (2.12) is the requirement that no free poles exist. Equations (2.10-2.12), coupled with (2.1-2.3), give

$$
\begin{aligned}
& \partial_{i} \partial_{i} u_{j}=0, \\
& \partial_{i} \partial_{i} \phi_{\mathrm{V}}=0, \\
& \partial_{i} \partial_{i} \phi_{\mathrm{M}}=0,
\end{aligned}
$$

or equivalently

$$
\begin{aligned}
& \nabla \cdot \nabla u=\nabla^{2} u=0, \\
& \nabla \cdot \nabla \phi_{\mathrm{V}}=\nabla^{2} \phi_{\mathrm{V}}=0, \\
& \nabla \cdot \nabla \phi_{\mathrm{M}}=\nabla^{2} \phi_{\mathrm{M}}=0 .
\end{aligned}
$$

These are simply Laplace's equations whose solutions in terms of rectangular or polar coordinates are readily found to be

$$
\begin{aligned}
& u_{z}=b\left(\frac{\theta}{2 \pi}\right)=\frac{b}{2 \pi} \tan ^{-1}\left(\frac{y}{x}\right), \\
& \phi_{\mathrm{V}}=\Gamma\left(\frac{\theta}{2 \pi}\right)=\frac{\Gamma}{2 \pi} \tan ^{-1}\left(\frac{y}{x}\right)
\end{aligned}
$$




$$
\phi_{\mathrm{M}}=I\left(\frac{\theta}{2 \pi}\right)=\frac{I}{2 \pi} \tan ^{-1}\left(\frac{y}{x}\right) .
$$

Equation (2.19) can be recognized to be the displacement field associated wilh a screw-type dislocation [7], whereas (2.20) and (2.21) are the potentials characteristic of vortices [8]. They are illustrated in Fig. 1 from which it is clear that the present treatment has been restricted to infinitely long line defects lying along the $z$ axis. The symbols $b, \Gamma$ and $I$ are termed the Burgers vector, circulation

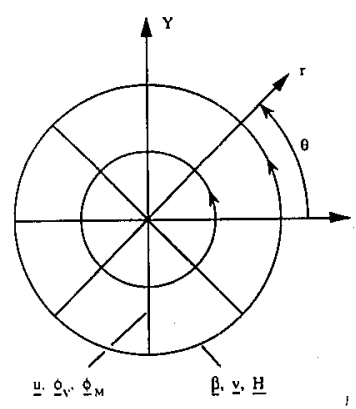

Fig. 1. Generalized characterization of infinite straight line defects that lie along the $z$ axis.

and current. These are invariant quantities that characterize their respective line defects. Combining (2.19-2.21), (2.7-2.9) and (2.1-2.3) yields

$$
\begin{gathered}
\sigma_{\theta z}=\frac{G b}{2 \pi r}, \\
P_{\theta}=\frac{\rho \Gamma}{2 \pi r}, \\
B_{\theta}=\frac{\mu I}{2 \pi r},
\end{gathered}
$$

or equivalently

$$
\begin{array}{ll}
\sigma_{x z}=-\frac{G b}{2 \pi} \frac{y}{\left(x^{2}+y^{2}\right)}, & \sigma_{y z}=\frac{G b}{2 \pi} \frac{x}{\left(x^{2}+y^{2}\right)}, \\
P_{x}=-\frac{\rho \Gamma}{2 \pi} \frac{y}{\left(x^{2}+y^{2}\right)}, & P_{y}=\frac{\rho \Gamma}{2 \pi} \frac{x}{\left(x^{2}+y^{2}\right)}, \\
B_{x}=-\frac{\mu I}{2 \pi} \frac{y}{\left(x^{2}+y^{2}\right)}, & B_{y}=\frac{\mu I}{2 \pi} \frac{x}{\left(x^{2}+y^{2}\right)},
\end{array}
$$

where (2.22-2.24) refer to polar coordinates, while (2.25-2.27) are in rectangular coordinates. For a screw-type dislocation $G$ in (2.25) and (2.22) replaces $c_{i j k \ell}$ in (2.7). In order not to obscure the inherent similarity between the three basic defects considered herein, the more complex edge-type dislocation has not been considered. Table I also shows that the ranks of the various quantities associated with solids is one higher than that for fluids or magnetostatics. In particular, $b_{i}$ is a vector whereas $\Gamma$ and $I$ are scalars. 


\section{The connection between defect densities and vector potential}

The total defect content $\sum b_{i}, \sum \Gamma$ and $\sum I$ within any closed path can be obtained from the following integrals:

$$
\begin{aligned}
& \sum b_{i}=\oint \beta_{j i} \mathrm{~d} x_{j}=\int \alpha_{k i} \mathrm{~d} S_{k}, \\
& \sum \Gamma=\oint v_{i} \mathrm{~d} x_{i}=\int w_{i} \mathrm{~d} S_{i}, \\
& \sum I=\oint H_{i} \mathrm{~d} x_{i}=\int J_{i} \mathrm{~d} S_{i},
\end{aligned}
$$

where Stoke's theorem has been used to convert the line integrals to surface integrals. The quantities $\alpha_{k i}, w_{i}$, and $J_{i}$ are commonly referred to as the dislocation density, vorticity and current density, respectively. They may be written as

$$
\begin{gathered}
\alpha_{k i}=\varepsilon_{k \ell j} \partial_{\ell} \beta_{j i}, \\
w_{i}=\varepsilon_{i j k} \partial_{j} v_{k}, \\
J_{i}=\varepsilon_{i j k} \partial_{j} H_{k},
\end{gathered}
$$

where $\varepsilon_{i j k}$ are permutation symbols, or equivalently as

$$
\begin{aligned}
& \boldsymbol{\alpha}=\nabla \times \boldsymbol{\beta}, \\
& \boldsymbol{w}=\nabla \times \boldsymbol{v}, \\
& \boldsymbol{J}=\nabla \times \boldsymbol{H} .
\end{aligned}
$$

If the line integral in (3.1) is taken about a single quantized dislocation, it is termed a Burgers circuit [9]. More generally, it may be referred to as a displacement circuit, whereas those given in (3.2) and (3.3) can be termed velocity and magnetic intensity circuits.

The condition that all of the line defects considered above form closed loops is satisfied by setting the divergences of all the defect densities equal to zero, or

$$
\begin{aligned}
& \partial_{i} \alpha_{i j}=0 \\
& \partial_{i} w_{i}=0 \\
& \partial_{i} J_{i}=0 .
\end{aligned}
$$

When applied to a single quantized defect, Eqs. (3.10-3.12), (2.7-2.9) and $(2.10-2.12)$ lead to the picture shown in Fig. 2. In terms of Eqs. (3.7-3.9), $\alpha$ may be considered to be the source of $\beta, w$ the source of $v$ and $J$ the source of $\boldsymbol{H}$.

The results given by $(2.22-2.24)$ can be arrived at in an entirely different manner by writing

$$
\begin{aligned}
& \sigma_{i j}=\varepsilon_{i k \ell} \partial_{k} A_{\ell j}, \\
& P_{k}=\varepsilon_{k \ell m} \partial_{\ell} A_{\mathrm{V} m}, \\
& B_{k}=\varepsilon_{k \ell m} \partial_{\ell} A_{\mathrm{Mm}},
\end{aligned}
$$



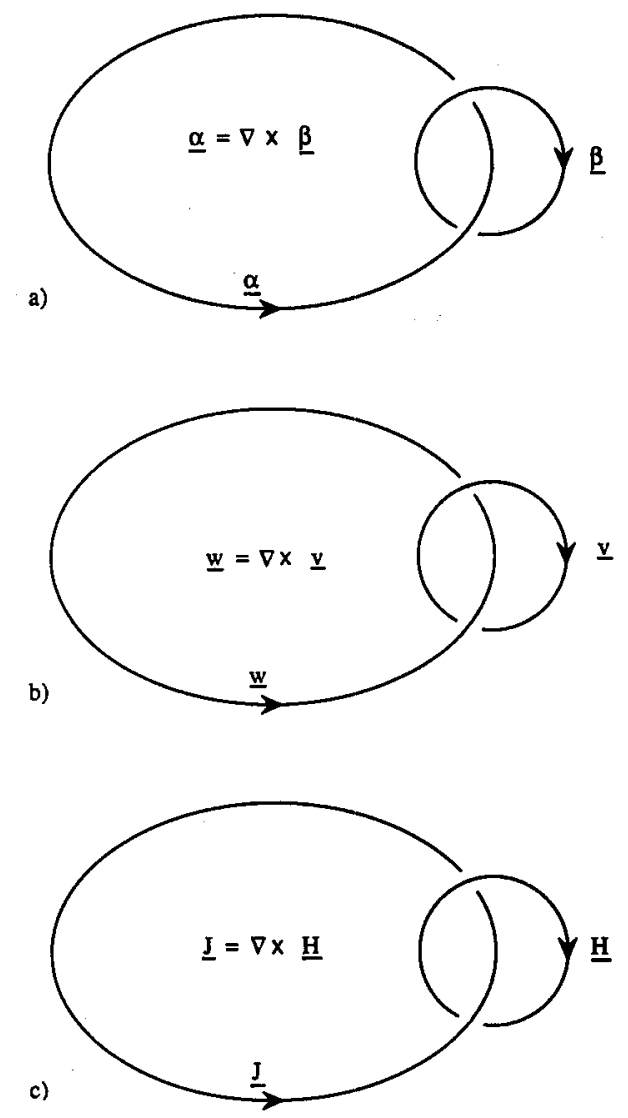

Fig. 2. (a) Dislocation, (b) vortex, and (c) current loops (ellipses) and their accompanying fields (circles).

or equivalently

$$
\begin{aligned}
& \boldsymbol{\sigma}=\nabla \times \boldsymbol{A}, \\
& \boldsymbol{P}=\nabla \times \boldsymbol{A}_{\mathrm{V}}, \\
& \boldsymbol{B}=\nabla \times \boldsymbol{A}_{\mathrm{M}},
\end{aligned}
$$

where $\boldsymbol{A}$ is a tensor potential, which is more commonly referred to as a stress function [3], while $\boldsymbol{A}_{\mathrm{V}}$ and $\boldsymbol{A}_{\mathrm{M}}$ are vector potentials associated with the fluid and magnetic vortices, respectively. That the vector and tensor potentials are actual physical quantities can be seen from the fact that they satisfy the following gauge transformation [10]

$$
\begin{aligned}
& \boldsymbol{A}^{\prime}=\boldsymbol{A}+\nabla \phi, \\
& \boldsymbol{A}_{\mathrm{V}}^{\prime}=\boldsymbol{A}_{\mathrm{V}}+\nabla \phi, \\
& \boldsymbol{A}_{\mathbf{M}}^{\prime}=\boldsymbol{A}_{\mathrm{M}}+\nabla \phi,
\end{aligned}
$$


where $\phi$ is some arbitrary scalar function. It is clear that since $\nabla \times \nabla \phi=0$, Eqs. (3.16-3.18) are left unaffected by this transformation. It follows from (2.7-2.9), (3.7-3.9) and (3.16-3.18) that

$$
\begin{aligned}
& \nabla \times \nabla \times \boldsymbol{A}=\nabla \times \boldsymbol{\sigma}=G \boldsymbol{\alpha}, \\
& \nabla \times \nabla \times \boldsymbol{A}_{\mathrm{V}}=\nabla \times \boldsymbol{P}=\boldsymbol{\rho w}, \\
& \nabla \times \nabla \times \boldsymbol{A}_{\mathrm{M}}=\nabla \times \boldsymbol{B}=\mu \boldsymbol{J} .
\end{aligned}
$$

Expanding the leftmost terms in the above relation [11], we obtain

$$
\begin{aligned}
& \nabla(\nabla \cdot \boldsymbol{A})-\nabla^{2} \boldsymbol{A}=G \boldsymbol{\alpha}, \\
& \nabla\left(\nabla \cdot \boldsymbol{A}_{\mathrm{V}}\right)-\nabla^{2} \boldsymbol{A}_{\mathrm{V}}=\rho \boldsymbol{w}, \\
& \nabla\left(\nabla \cdot \boldsymbol{A}_{\mathrm{M}}\right)-\nabla^{2} \boldsymbol{A}_{\mathbf{M}}=\mu \boldsymbol{J} .
\end{aligned}
$$

Imposing the following gauge conditions on the above

$$
\begin{aligned}
& \nabla \cdot \boldsymbol{A}=0, \\
& \nabla \cdot \boldsymbol{A}_{\mathbf{V}}=0, \\
& \nabla \cdot \boldsymbol{A}_{\mathbf{M}}=0,
\end{aligned}
$$

leads to

$$
\begin{aligned}
& \nabla^{2} A=-G \alpha \\
& \nabla^{2} A_{V}=-\rho w \\
& \nabla^{2} A_{M}=-\mu J
\end{aligned}
$$

The solutions to these Poisson type equations are readily found to be

$$
\begin{aligned}
& \boldsymbol{A}=\frac{G}{4 \pi} \int \frac{\boldsymbol{\alpha}}{R} \mathrm{~d} V^{\prime}, \\
& \boldsymbol{A}_{\mathrm{V}}=\frac{\rho}{4 \pi} \int \frac{\boldsymbol{w}}{R} \mathrm{~d} V^{\prime}, \\
& \boldsymbol{A}_{\mathrm{M}}=\frac{\mu}{4 \pi} \int \frac{\boldsymbol{J}}{R} \mathrm{~d} V^{\prime},
\end{aligned}
$$

where the position vector $R=r-r^{\prime}$. The above volume integrals can be readily integrated for infinitely long line defects lying along the $z$ axis and give [12]

$$
\begin{aligned}
& A_{z z}=-\frac{G b}{2 \pi} \ln r+\text { const } \\
& A_{V z}=-\frac{\rho \Gamma}{2 \pi} \ln r+\text { const, } \\
& A_{M z}=-\frac{\mu I}{2 \pi} \ln r+\text { const. }
\end{aligned}
$$

When the above are used in conjuction with Eqs. (3.13-3.15), results for $\sigma_{\theta z}$, $P_{\theta}$ and $B_{\theta}$ identical to those given by $(2.22-2.24)$ are obtained. Although similar in principle, the methods used to determine the tensor potential for an infinite straight edge-type dislocation are somewhat more involved [3].

The results obtained thus far may be represented pictorially as shown in Figs. 2 and 3. Figure 2, in particular, shows how the various defects are related to their fields in accordance with (3.7-3.9). The fact that each forms closed loops satisfies the divergence conditions of (3.10-3.12) and (2.10-2.12). In a similar manner, 

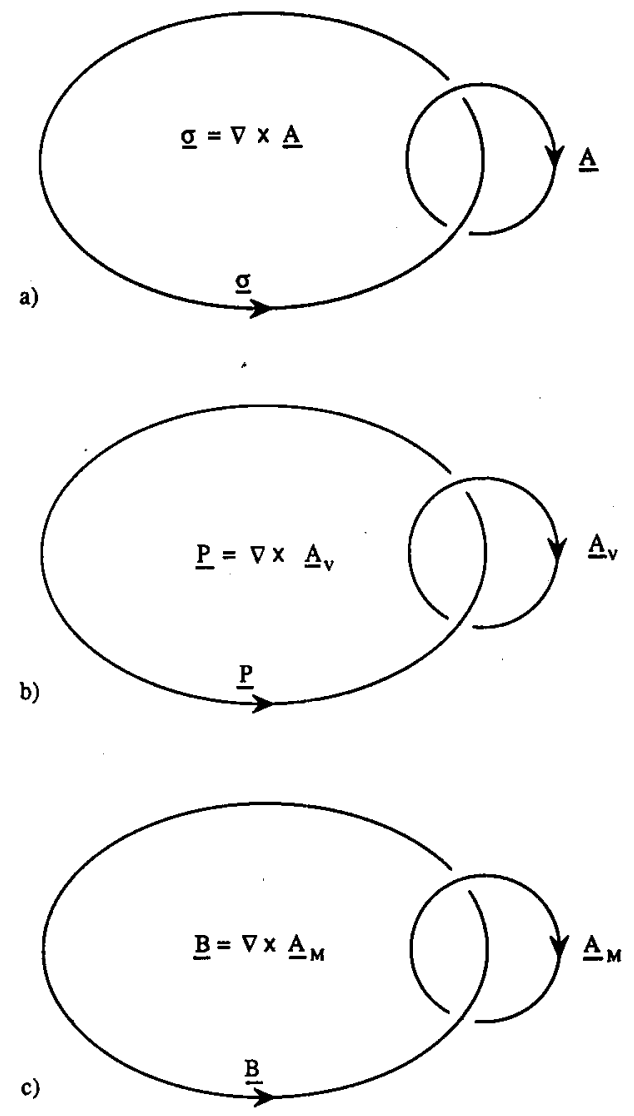

Fig. 3. Fields (ellipses) associated with (a) dislocation, (b) vortex, and (c) current loops and their accompanying potentials (circles).

Fig. 3 demonstrates how the vector potentials are related to their corresponding fields according to (3.16-3.18). The closed loop nature of each is once again guaranteed by the vanishing of the divergences given in (3.28-3.30) and (2.10-2.12).

The remarkable similarity between Figs. 2 and 3 suggests that the former, in view of (3.7-3.9), can be expressed in terms of the following gauge transformations, similar to (3.19-3.21):

$$
\begin{aligned}
& \boldsymbol{\beta}^{\prime}=\boldsymbol{\beta}+\nabla \phi, \\
& \boldsymbol{v}^{\prime}=\boldsymbol{v}+\nabla \phi, \\
& \boldsymbol{H}^{\prime}=\boldsymbol{H}+\nabla \phi,
\end{aligned}
$$

where (3.10-3.12) now become the gauge conditions for those transformations. Equation (3.40) was first recognized by Golebiewska-Lasota [13] who drew her analogy from (3.21). 
Comparison of Figs. 2 and 3 also allows us to write the following relations along the lines given by (3.1-3.3)

$$
\begin{aligned}
& \sum \Phi=\oint \boldsymbol{A} \cdot \mathrm{d} \boldsymbol{x}=\int \boldsymbol{\sigma} \cdot \mathrm{d} \boldsymbol{S}, \\
& \sum \Phi_{\mathrm{V}}=\oint A_{\mathrm{V}} \cdot d x=\int \boldsymbol{P} \cdot \mathrm{d} \boldsymbol{S}, \\
& \sum \Phi_{\mathrm{M}}=\oint A_{\mathrm{M}} \cdot \mathrm{d} \boldsymbol{x}=\int \boldsymbol{B} \cdot \mathrm{d} \boldsymbol{S},
\end{aligned}
$$

where $\sum \Phi$ and $\sum \Phi_{\mathrm{V}}$ may be viewed as the total stress and momentum flux within the circuit in analogy with the definition of magnetic flux, $\sum \Phi_{M}$ [14]. These three quantities correspond to $\sum b, \sum \Gamma$ and $\sum I$, respectively, in (3.1-3.3).

\section{Characteristics of line defects}

The self energy per unit length of the various line defects considered thus far can be written as [ 6 ]

$$
\begin{aligned}
& E_{\mathrm{DS}}=\frac{G b^{2}}{4 \pi} \ln \left(\frac{r_{1}}{r_{0}}\right), \\
& E_{\mathrm{VS}}=\frac{\rho \Gamma^{2}}{4 \pi} \ln \left(\frac{r_{1}}{r_{0}}\right), \\
& E_{\mathrm{MS}}=\frac{\mu I^{2}}{4 \pi} \ln \left(\frac{r_{1}}{r_{0}}\right),
\end{aligned}
$$

where $r_{1}$ is on the order of the body dimensions containing the defect, while $r_{0}$ is the radius of the defect core. $E_{\mathrm{DS}}$ consists entirely of elastic strain energy, $E_{\mathrm{VS}}$ of kinetic energy and $E_{\mathrm{MS}}$ of magnetic energy. On the other hand, the interaction energies between a pair of parallel line defects of opposite sign and spacing $r$ are given as follows:

$$
\begin{aligned}
& E_{\mathrm{DI}}=-\frac{G b^{2}}{2 \pi} \ln \left(\frac{r_{1}}{r}\right), \\
& E_{\mathrm{VI}}=-\frac{\rho \Gamma^{2}}{2 \pi} \ln \left(\frac{r_{1}}{r}\right), \\
& E_{\mathrm{MI}}=-\frac{\mu I^{2}}{2 \pi} \ln \left(\frac{r_{1}}{r}\right) .
\end{aligned}
$$

If the three defects treated thus far are placed in external fields $\sigma_{i j}, P_{i}$, and $B_{i}$, they experience the following forces per unit length:

$$
\begin{aligned}
& F_{\mathrm{D} n}=-\varepsilon_{n m j} t_{m} \sigma_{i j} b_{i}, \\
& F_{\mathrm{V} n}=-\varepsilon_{n m i} t_{m} P_{i} \Gamma, \\
& F_{\mathrm{Mn}}=-\varepsilon_{n m i} t_{m} B_{i} I,
\end{aligned}
$$

or equivalently

$$
F_{\mathrm{D}}=t \times(\sigma \cdot b)
$$




$$
\begin{aligned}
& \boldsymbol{F}_{\mathrm{V}}=\Gamma(t \times P), \\
& \boldsymbol{F}_{\mathrm{M}}=I(t \times B),
\end{aligned}
$$

where $t$ is a unit vector tangent to the dislocation line. Equation (4.10) is commonly referred to as the Peach-Koehler force on a dislocation [15]. $\boldsymbol{F}_{\mathbf{V}}$, on the other hand, is the well-known force arising from the Magnus effect responsible for lift in airfoils [16]. The force $\boldsymbol{F}_{\mathbf{M}}$ is termed Ampère's law, which is also the Lorentz force [14].

The self energies of circular loops can be readily obtained from (4.1-4.3) by replacing $r_{1}$ therein by $R$, the radius of the loop, followed by multiplication of each expression by $2 \pi R[6]$.

\section{Irrotational fields in terms of surface line defect distributions}

Somigliana's identity can be used to describe the displacement $u$ at point $x^{\prime}$ within a finite region of an infinite body in terms of the following surface integral along the boundary [17]

$$
u_{i}\left(x^{\prime}\right)=\int\left[t_{j}(x) U_{i j}\left(x^{\prime}-x\right)-u_{j}(x) T_{i j}\left(x^{\prime}-x\right)\right] \mathrm{d} S,
$$

where $U_{i j}\left(x^{\prime}-x\right)$ is the $i$-th component of the displacement at $x^{\prime}$ produced by a unit force applied in the $j$-th direction at $x$, the so-called Green's tensor function. The quantities $t_{j}(x)$ and $T_{i j}\left(x^{\prime}-x\right)$ are the boundary tractions corresponding to the displacements $u_{j}(x)$ and $u_{i j}$, respectively. It is a relatively simple matter to extend (5.1) so as to include the cases of fluid flow and magnetostatics as follows

$$
\begin{aligned}
& \phi_{\mathrm{V}}\left(x^{\prime}\right)=\int\left[t_{\mathrm{V}}(x) \Phi_{\mathrm{V}}\left(x^{\prime}-x\right)-\phi_{\mathrm{V}}(x) T_{\mathrm{V}}\left(x^{\prime}-x\right)\right] \mathrm{d} S, \\
& \phi_{\mathrm{M}}\left(x^{\prime}\right)=\int\left[t_{\mathrm{M}}(x) \Phi_{\mathrm{M}}\left(x^{\prime}-x\right)-\phi_{\mathrm{M}}(x) T_{\mathrm{M}}\left(x^{\prime}-x\right)\right] \mathrm{d} S,
\end{aligned}
$$

where, in the case of $(5.2), \Phi_{\mathrm{V}}\left(x^{\prime}-x\right)$ is the velocity potential at $x^{\prime}$ produced by a unit source at $x$ on the boundary. The quantities $t_{\mathrm{V}}(x)$ and $T_{\mathrm{V}}\left(x^{\prime}-x\right)$ are the boundary sources corresponding to the potentials $\Phi_{\mathrm{V}}(x)$ and $T_{\mathrm{V}}\left(x^{\prime}-x\right)$, respectively. The terms in (5.3) are accounted for in a similar manner.

When the tractions, $t_{j}(x)$, across the boundary are made continuous, (5.1) reduces to $[18,19]$

$$
u_{i}\left(x^{\prime}\right)=\int b_{i}(x) T_{i j}\left(x^{\prime}-x\right) \mathrm{d} S .
$$

The above equation can now be viewed in terms of a surface dislocation whose Burgers vector $b_{i}(x)$ varies as a function of position, $x$, on the surface. Such an entity has been termed a Somigliana dislocation [20]. Similarly, when the sources $t_{\mathrm{V}}(x)$ and $t_{\mathrm{M}}(x)$ are continuous across the boundary, (5.2) and (5.3) reduce to

$$
\begin{aligned}
& \phi_{\mathrm{V}}\left(x^{\prime}\right)=\int \Gamma(x) T_{\mathrm{V}}\left(x^{\prime}-x\right) \mathrm{d} S, \\
& \phi_{\mathrm{M}}\left(x^{\prime}\right)=\int I(x) T_{\mathrm{M}}\left(x^{\prime}-x\right) \mathrm{d} S,
\end{aligned}
$$


where for the last three equations

$$
\begin{gathered}
b_{i}(x)=u_{i}^{-}(x)-u_{i}^{+}(x), \\
\Gamma(x)=\phi_{\mathrm{V}}^{-}(x)-\phi_{\mathrm{V}}^{+}(x), \\
I(x)=\phi_{\mathrm{M}}^{-}(x)-\phi_{\mathrm{M}}^{+}(x),
\end{gathered}
$$

where the plus and minus superscripts refer to opposite faces of the boundary. For completeness, we may also write

$$
\begin{aligned}
& t_{j}=\sigma_{j i} n_{i}, \\
& t_{\mathrm{V}}=P_{i} n_{i}, \\
& t_{\mathrm{M}}=B_{i} n_{i},
\end{aligned}
$$

where $n_{i}$ is the unit normal to the boundary. Eshelby $[20,21]$, on the basis of (5.4), has concluded that knowledge of $b_{i}(x)$ completely determines the state of deformation within the body. Since a Somigliana dislocation can be represented in terms of a continous distribution of elementary line dislocations [22], it follows that any state of deformation can be expressed in terms of such distributions. Dislocations comprising such arrays have been termed surface dislocations $[4,5]$ and provide a powerful method for the solution of any type of boundary value problem.

Extending Eshelby's arguments to fluids and magnetostatics, it follows from (5.5 and 5.6) that a knowledge of $\Gamma(x)$ and $I(x)$ completely determines the state of fluid flow and magnetization, respectively, within a finite body. Carrying the analogy still further, it can be concluded that any state of fluid flow or magnetization within a given boundary can be represented in terms of some unique distribution of surface vortices. This has already been demonstrated in the case of fluids [6].

Utilization of the surface dislocation technique is most easily seen by reference to Fig. 4a, which shows a uniform array of surface vortices (dotted symbols) of opposite sign on adjacent sides of a finite rectangular body. The vortices are parallel to the $z$ axis and are of infinite length. Consider first the limiting case where the body is infinitely long and of width $2 c$. The dislocations, which have Burgers vectors $b$ and spacing $h$, in Fig. 4a may now be thought of as continuously distributed with a constant dislocation density of $b / h$. Utilizing $(2.25)$ the $\sigma_{y z}$ component of stress for this array may be written as

$$
\sigma_{y z}=\frac{G b}{2 \pi h} \int_{-\alpha}^{+\alpha} \frac{(x+c) \mathrm{d} y^{\prime}}{(x+c)^{2}+\left(y+y^{\prime}\right)^{2}}-\frac{G b}{2 \pi h} \int_{-\alpha}^{+\alpha} \frac{(x-c) \mathrm{d} y^{\prime}}{(x-c)^{2}+\left(y+y^{\prime}\right)^{2}}
$$

where the first integral corresponds to the stress field of the leftmost wall in Fig. 4a, while the second integral pertains to the rightmost wall. Upon integration, we obtain

$$
\sigma_{y z}=\frac{G b}{2 \pi h}\left[\left.\tan ^{-1}\left(\frac{y+y^{\prime}}{x+c}\right)\right|_{-\infty} ^{+\infty}-\left.\tan ^{-1}\left(\frac{y+y^{\prime}}{x-c}\right)\right|_{-\infty} ^{+\infty}\right]
$$




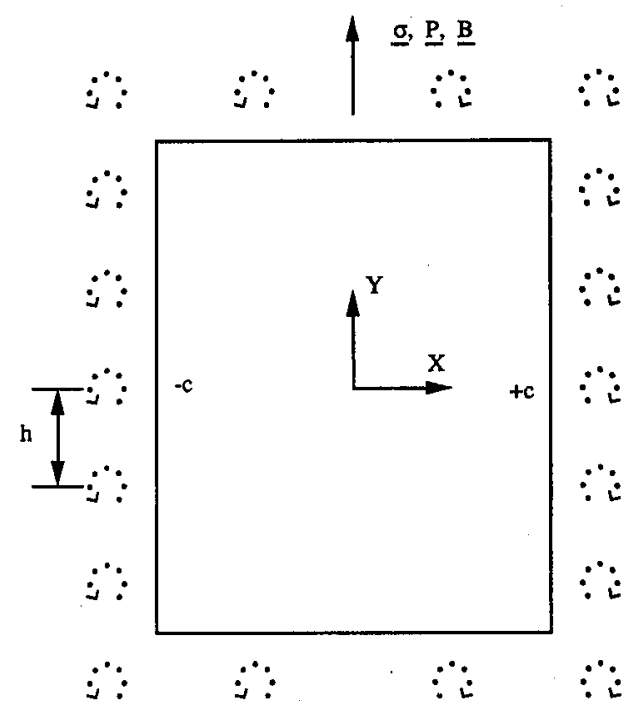

a)

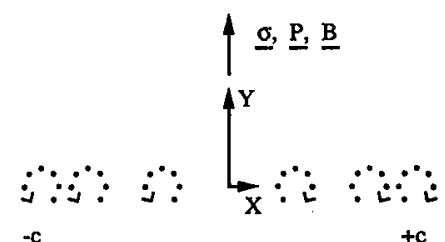

b)

Fig. 4. (a) Two-dimensional, (b) one-dimensional arrangement of line defects.

which gives

$$
\begin{array}{ll}
\sigma_{y z}=\frac{G b}{h}=-\sigma, & -c<x<+c, \\
\sigma_{y z}=0, & -c>x>+c .
\end{array}
$$

A similar calculation for $\sigma_{x z}$ shows that it vanishes everywhere. We conclude that Fig. $4 \mathrm{a}$ in the case of dislocations corresponds to a stressed body under antiplane strain.

When the line defects in Fig. $4 \mathrm{a}$ are taken to be fluid or magnetic vortices, (2.26) and (2.27) can be used to write the counterparts of (5.13) as follows:

$$
\begin{aligned}
& P_{y}=\frac{\rho \Gamma}{2 \pi h} \int_{-\infty}^{+\infty} \frac{(x+c) \mathrm{d} y^{\prime}}{(x+c)^{2}+\left(y+y^{\prime}\right)^{2}}-\frac{\rho \Gamma}{2 \pi h} \int_{-\infty}^{+\infty} \frac{(x-c) \mathrm{d} y^{\prime}}{(x-c)^{2}+\left(y+y^{\prime}\right)^{2}} \\
& B_{y}=\frac{\mu I}{2 \pi h} \int_{-\infty}^{+\infty} \frac{(x+c) \mathrm{d} y^{\prime}}{(x+c)^{2}+\left(y+y^{\prime}\right)^{2}}-\frac{\mu I}{2 \pi h} \int_{-\infty}^{+\infty} \frac{(x-c) \mathrm{d} y^{\prime}}{(x-c)^{2}+\left(y+y^{\prime}\right)^{2}}
\end{aligned}
$$

which, upon integration, gives

$$
\begin{array}{ll}
P_{y}=-\frac{\rho \Gamma}{h}=P, & -c<x<+c \\
P_{y}=0, & -c>x>+c,
\end{array}
$$




$$
\begin{array}{ll}
B_{y}=\frac{\mu I}{h}=B, & -c<x<+c, \\
B_{y}=0, & -c>x>+c,
\end{array}
$$

in analogy with (5.15). Similarly, $P_{x}$ and $B_{x}$ vanish everywhere. Physically, (5.18) represents uniform irrotational flow through an infinitely long channel. On the other hand, (5.19) corresponds to the uniform magnetization within an infinitely long solenoid.

When the length of the body in Fig. $4 \mathrm{a}$ becomes finite, the end effects become important and analytic solutions for the various fields within the body are not possible. Under these conditions, numerical techniques are readily available [5]. It becomes instructive now, however, to consider another limiting case wherein the array of Fig. 4a is made planar, as depicted in Fig. 4b. Furthermore, it is assumed that $\sigma_{y z}=\sigma$ for $y=0$ between $+c$ and $-c$. This condition is met by writing

$$
\sigma_{y z}(x, 0)=\frac{G b}{2 \pi} \quad \int_{-c}^{+c} \frac{f\left(x^{\prime}\right) \mathrm{d} x^{\prime}}{x^{\prime}-x}=\sigma,
$$

where the barred integral sign denotes the Cauchy principal value. Its solution yields the following dislocation distribution function [5, 23]

$$
f(x)=\frac{2 \sigma}{G b} \frac{x}{\left(c^{2}-x^{2}\right)^{1 / 2}}
$$

where

$$
\int_{0}^{a} f(x) \mathrm{d} x=N
$$

$N$ being the total number of dislocations with Burgers vector $b$ between 0 and $a$.

When the line defects in Fig. $4 \mathrm{~b}$ are taken to be fluid and magnetic vortices, (5.20) becomes

$$
\begin{aligned}
& P_{y}(x, 0)=\frac{\rho \Gamma}{2 \pi} \quad \int_{-c}^{+c} \frac{f\left(x^{\prime}\right) \mathrm{d} x^{\prime}}{x^{\prime}-x}=P, \\
& B_{y}(x, 0)=\frac{\mu I}{2 \pi} \quad \int_{-c}^{+c} \frac{f\left(x^{\prime}\right) \mathrm{d} x^{\prime}}{x^{\prime}-x}=B,
\end{aligned}
$$

from which we obtain, analogous to (5.21)

$$
\begin{aligned}
& f(x)=\frac{2 P}{\rho \Gamma} \frac{x}{\left(c^{2}-x^{2}\right)^{1 / 2}}, \\
& f(x)=\frac{2 B}{\mu I} \frac{x}{\left(c^{2}-x^{2}\right)^{1 / 2}},
\end{aligned}
$$

where (5.22) again holds; however, $N$ now represents the number of fluid and magnetic vortices with circulation $\Gamma$ and current $I$, respectively between 0 and a.

Suppose the fields $-\sigma,-P$ and $-B$ are superimposed on Fig. 4a. This transforms (5.15), (5.18) and (5.19) into

$$
\begin{array}{ll}
\sigma_{y z}=0, & -c<x<+c, \\
\sigma_{y z}=-\frac{G b}{h}=-\sigma, & -c>x>+c, \\
P_{y}=0, & -c<x<+c, \\
P_{y}=-\frac{\rho \Gamma}{h}=-P, & -c>x>+c,
\end{array}
$$




$$
\begin{array}{ll}
B_{y}=0, & -c<x<+c, \\
B_{y}=-\frac{\mu I}{h}=-B, & -c>x>+c,
\end{array}
$$

where (5.27) corresponds to a stressed hole [24], Eq. (5.28) to flow past a rigid body, and (5.29) to the expulsion of magnetic flux from a type I superconductor; i.e., the Meissner effect [25].

If, in a similar manner, the fields $-\sigma,-P$ and $-B$ are superimposed on Fig. $4 \mathrm{~b},(5.20),(5.23)$ and $(5.24)$ are transformed into

$$
\begin{gathered}
\sigma_{y z}(x, 0)=0, \\
P_{y}(x, 0)=0, \\
B_{y}(x, 0)=0 .
\end{gathered}
$$

Equation (5.30) corresponds to an antiplane shear or mode III crack [5, 23], Eq. (5.31) to a momentum concentration at some obstacle to fluid flow [26], while Eq. (5.32) represents the magnetic flux concentration about a thin type I superconductor situated within a magnetic field.

All of the fields associated with Fig. 4 are termed irrotational. This means that there are no defects within the interior of the body so that $\alpha, w$ and $J$ given by (3.7-3.9) vanish therein. For example, the field produced by $\sigma$ generates purely elastic distortions.

\section{Rotational fields}

As soon as a line defect is introduced into an irrotational field, it becomes rotational. One of the simplest types of arrangement is the dipole shown by solid symbols in Fig. 5. Under equilibrium conditions, the extension of the dipole can be written as [6]

$$
\begin{aligned}
& d_{\mathrm{D}}=\frac{G b}{2 \pi \sigma}, \\
& d_{\mathrm{V}}=\frac{\rho \Gamma}{2 \pi P}, \\
& d_{\mathrm{M}}=\frac{\mu I}{2 \pi B} .
\end{aligned}
$$

When dislocations are involved, as in (6.1), the result is plastic deformation. Formation of defect pairs leads to a reduction of $\sigma, P$ and $\boldsymbol{B}$ in their immediate vicinity, resulting in a decrease of strain, kinetic and magnetic energies, respectively. It also follows that the introduction of defects into a uniform field causes it to become more chaotic and may be viewed as the basic ingredient of turbulence [26]. This seems to be borne out by studies with helium II [27]. 


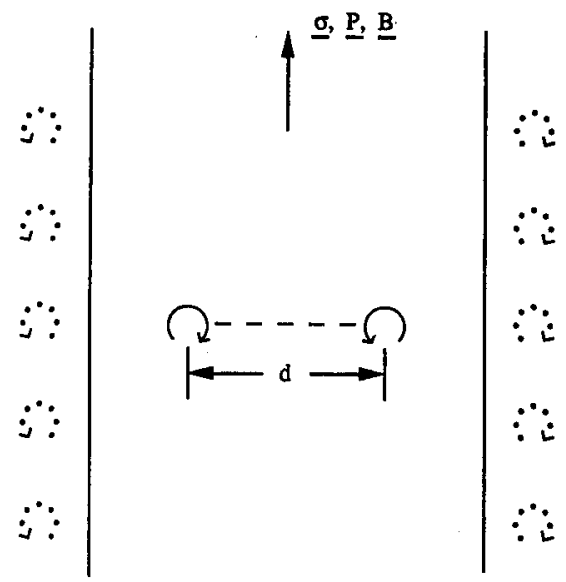

Fig. 5. Rotational flow due to the formation of a dipole.

\section{Discussion}

Dislocations, fluid vortices, as well as magnetic vortices have all been shown to be mathematically identical with one another. In essence, they fall into the category of line defects, interacting with one another, possessing a line tension, a self energy and responding to an external field. Most important of all, when arranged into certain arrays, they can be used to represent any type of irrotational (defect-free) and rotational (defect-present) fields. It is these latter fields that are the most important and at the same time present the most perplexing and difficult problems. Their understanding lies at the heart of resolving the concept of turbulence. In solids, turbulent behavior manifests itself during plastic deformation and workhardening, in liquids by erratic and unpredictable flow at high Reynolds numbers, while in large magnetic fields it appears in the form of energy dissipating eddy currents. By presenting a unified and detailed treatment of this broad class of defects, it is hoped that a foundation has been laid for a clearer understanding of these more difficult problems. Lastly, it is hoped that the present effort will spur the search for line defects in an even broader class of fields, including gravitation.

\section{References}

[1] J.M. Burgers, in: Proc. K. Ned. Akad. Wet. 42, 293 (1939).

[2] E. Kröner, Erg. Angew. Math. 5, 1 (1958).

[3] R. de Wit, in: Solid State Physics, Eds. F. Seitz, D. Turnbull, Vol. 10, Academic Press, New York 1960, p. 249.

[4] M.J. Marcinkowski, Unified Theory of the Mechanical Behavior of Matter, Wiley, New York 1979.

[5] K. Jagannadham, M.J. Marcinkowski, Unified Theory of Fracture, Trans. Tech. Publications, Aedermannsdorf (Switzerland) 1983.

[6] M.J. Marcinkowski, Phys. Status Solidi B 152, 9 (1989). 
[7] A.H. Cottrell, Dislocations and Plastic Flow in Crystals, Clarendon Press, Oxford 1953.

[8] G.K. Batchelor, An Introduction to Fluid Dynamics, University Press, Cambridge 1970.

[9] E. Kröner, in: Vorlesungen über Theoretische Physik, Ed. A. Sommerfeld, Vol. 2, 5th ed., Chap. 9, Akademische Verlagsgesellschaft Geest and Portig, Leipzig 1964, p. 310 .

[10] L.D. Landau, E.M. Lifshitz, The Classical Theory of Fields, Pergamon Press, Oxford 1975.

[11] H. Lass, Vector and Tensor Analysis, McGraw-Hill, New York 1950.

[12] R.K. Wangsness, Electromagnetic Fields, Wiley, New York 1979.

[13] A.A. Golebiewska-Lasota, Int. J. Eng. Sci. 17, 329 (1979).

[14] R.G. Fowler, Introduction to Electric Theory, Addison-Wesley, Cambridge, Massachusetts 1953.

[15] M. Peach, J.S. Koehler, Phys. Rev. 80, 436 (1950).

[16] L. Prandtl, Essentials of Fluid Dynamics, Hafner, New York 1952.

[17] A.E.H. Love, A Treatise on the Mathematical Theory of Elasticity; Dover Publications, New York 1944.

[18] N.J. Altiero, S.D. Gavazza, J. Elast. 10, 1 (1980).

[19] M. Maiti, J. Elast. 9, 425 (1979).

[20] J.D. Eshelby, in: Solid State Physics, Eds. F. Seitz, D. Turnbull, Vol. 3, Academic Press, New York 1956, p. 79.

[21] J.D. Eshelby, in: Dislocations in Solids, Ed. F.R.N. Nabarro, Vol. 3, North-Holland, Amsterdam 1979, p. 167.

[22] M. Maiti, B. Das, S.S. Palit, J. Elast. 6, 429 (1976).

[23] R.W. Lardner, Mathematical Theory of Dislocations and Fracture, University of Toronto Press, Toronto 1974.

[24] M.J. Marcinkowski, E.S.P. Das, Int. J. Fract. 10, 181 (1974).

[25] C. Kittel, Introduction to Solid State Physics, Wiley, New York 1953.

[26] M.J.Marcinkowski, Philos. Mag. A 62, 363 (1990).

[27] R.J. Donnelly, Sci. Am. (USA) 259, 100 (1988). 